\title{
A CASE STUDY ABOUT FACEBOOK USAGE FOR VISUALLY IMPAIRED USING THE ANDROID PLATFORMS
}

\author{
Arda Uslu ${ }^{1}$ and Pinar Sarisaray-Boluk ${ }^{2}$ \\ ${ }^{1}$ Bahcesehir University, Computer Engineering \\ ${ }^{2}$ Bahcesehir University, Software Engineering \\ Istanbul, Turkey
}

\begin{abstract}
The use of social media plays an important role in our lives. It is crucial that visually impaired (VI) can likewise take part in various social media platforms. In this paper, we tried to discover the problems and interactions faced by VI on Facebook with the help of tasks that are prepared beforehand. We, researchers helped the participant to pass the steps required to complete these predetermined tasks if the participant encountered a problem. According to the first factor, the results are displayed in terms of points attributed to the steps. Secondly, the time required to complete the task is checked. The third factor is if the participant had already known it before or not. As a result, findings in terms of usability and accessibility are discussed. Hence, we tried to find out what can be done to address these issues.
\end{abstract}

\section{KEYWORDS}

Visually Impaired, Accessibility, Usability, Facebook

\section{INTRODUCTION}

Social Network Sites (SNS) are the most visited websites in the world. As of October 2018, the social networks by number of monthly active users are; Facebook (2.234 billion), YouTube (1.9 billion), WhatsApp (1.500 billion), Facebook Messenger (1.300 billion), Instagram (1 billion) (Global social media ranking 2019. (n.d.)). These numbers are expected to grow even further especially with the robust growth of the population in developing countries. Faster internet access and a boost in mobile phone sales with economic development will inevitably consolidate this trend. Naturally, these numbers also include users with special needs. In today's modern world it is an obligation to integrate them from all perspectives. In an increasingly equal and just society, VI deserves to be treated as equal as possible in comparison to people who do not have special needs. Accessibility of disabled users began to be a major issue in the last decade. To overcome these challenges, Facebook (FB) founded its Accessibility Team in July of 2011.

In this paper, research is carried out with the help of a VI person on FB. Prior research shows that visually impaired people have difficulty in accessing it (Barbara Leporini et al., 2012; Bavani Ramayah et al., 2013; Berglind Smaradottir et al., 2017; Brian M. Celusnak, 2016; Cynthia L. Bennett et al., 2018; Haley MacLeod et al., 2017; Rakesh Babu \& Rahul Singh, 2013; Rakesh Babu, 2014; Ramayah, B. et al., 2012). There is a lot of research regarding this phenomenon. Wu and Adamic (2014) had an extensive study about FB usage that benefits from huge chunk of FB data. They utilize big data techniques to gain information about VI behaviors on FB. However, it's only on iOS devices. Most of the research about visually challenged people's use of social media are on iOS devices (Barbara Leporini et al., 2012; Berglind Smaradottir et al., 2017; Brian M. Celusnak, 2016; Cynthia L. Bennett et al., 2018; Haley MacLeod et al., 2017; Joao Guerreiro \& Daniel Gonçalves, 2013; Shaomei Wu et al., 2017; Shaomei Wu \& Lada A. Adamic., 2014; Shi Qiu et al., 2015; Violeta Voykinska et al., 2016). Android devices are so rare (Aditya Vashistha et al., 2015; Andre Rodrigues et al., 2015; Cynthia L. Bennett et al., 2018). From those, Vashistha et al., (2015) made a detailed study about the usage of social media in rural and peri-rural parts of India. In it, the only thing about Android is just the brief mention of Talkback screen reader. André Rodrigues et al. (2015) mentioned the smartphone adoption process and general 
functions of smartphones with no comment about social media practices (Andre Rodrigues et al., 2015). Cynthia L. Bennett et al., (2018) made a research about the photo-taking habits of VI by using audio and video recordings just like our study. Roughly half of the participants in that study are Android users. There is a lack of research in Android devices although they are widely used throughout the world as well. We are trying to fill in this gap. In this paper, we aim to test the usability of FB functions with the help of a visually impaired person in an Android platform. There are 6 specific tasks (Change Language, Change Profile, Edit Username, Ignore Message, Unignore Message, Activate Status) given to test her on Facebook Messenger.

The rest of the paper is organized as follows: Section II gives our methodology. Section III presents the results and discussion. The paper concludes in Section IV concerning future work.

\section{METHODOLOGY}

Before the interview and the scenario section that followed, we had already prepared a document that includes necessary steps to complete certain functions on FB. Those functions were determined beforehand. Unlike, the works (Brian Wentz \& Jonathan Lazar, (2011); Shi Qiu et al., (2015)) that have more basic operations such as read and post, check-in, create an account, $\log$ in to an account we tested operations like ignore/unignore message, change the language settings. Think aloud observation method in Rakesh Babu \& Rahul Singh, (2013) was not chosen for this method. It was thought to lengthen the natural time the participant would otherwise spend using that method. The online survey used by Meredith Ringel Morris et al. (2016) on Twitter was not an option because of the lack of suitable participants.

This work is examined and confirmed by the Ethical Committee of the Bahcesehir University for ethical standards. This is realized for any kind of experiments that require human interaction in any discipline. The questionnaires and the detailed explanation of the project have been added to the authorization form. All the participants accepted to be part of the project. They accepted that they will not take any kind of benefits as a result of this study. They also have the right to give up whenever they want. We read the conditions each time before we started our interviews and we started as soon as they accepted it. Their acceptance speeches are kept in my voice recorder device (Sony IC Recorder ICD-P110) with the other parts of the interviews. Sometimes we took a video to see which buttons they pressed. Few of them asked questions to clarify the things they wanted to know. We did not disclose their identity and we signed a paper that conditions us to obey the rules determined by the University Ethical Committee. We searched lots of candidates in a Visually Impaired Association Headquarters. However, it was not possible to find long-term FB users. Some of them were not using it for a long time. Their stay was also not permanent. That's why we found another participant from the disabled unit of the university.

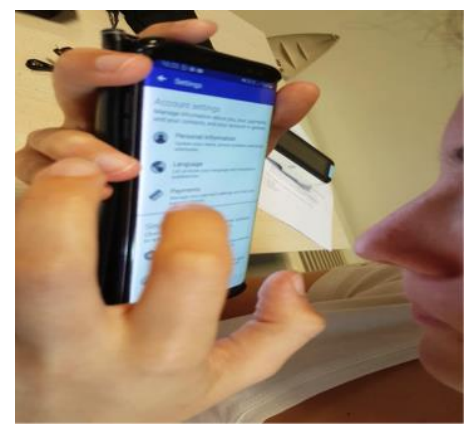

Figure 1. While the participant of the experiment was trying to perform the duties according to the scenario

Our participant is a female of 22 years old. She studies English Language Teaching (ELT) in the university that we conducted this research. She was prematurely born of 5.5 months. That made her visually impaired. She has a $\% 20$ vision in one eye. Her other eye is slightly worse even though she cannot remember the exact value. She has no difficulty in detecting light, colors, etc. She uses Facebook for almost 10 years in both English and Turkish. She does not recall using computer web browsers to access FB since 2016. She also uses Instagram. She never used Twitter. When she registered on for Facebook the first time, she does not remember that she had a problem with the CAPTCHA (Completely Automated Public Turing test to tell Computers and 
Humans Apart) that existed in those days. She said she could see when she opens her eyes wide. However, she needs to come near a little bit like the VI person who takes photos (Cynthia L. Bennett et al., 2018).

To make a usability analysis of FB functions for an impaired person, we used 6 different tasks which include Change Language, Change Profile, Edit Username, Ignore Message, Unignore Message, Activate Status. All of the tasks are firstly divided into main steps and assigned a success percentage value for each step. After identifying these steps, the participant tried to finish her tasks, which was shown in Figure 1.

\section{Change Language}

Add the language if it is not on the list. $(5 * 10=\% 50)$

Settings -> General Management -> Language and input -> Language -> Add Language.

If it is already on the list.

i. Click Edit. (\%15)

ii. Click arrows to move it at the top of the list. (\%15)

iii. Check Facebook for change. (\%20)

Social->Facebook

\section{Change Profile}

How to change profile picture (DP) in the Facebook messenger app.

1. Click on "Add to your story" with the + icon. (\%20)

2. Take a photo or go to the Gallery. (\%20 for Gallery)

3. Select the picture you want to add. (\%20)

4. Press "->" icon. (Your story icon) (\%20)

5. In the send to screen check the box to make it easy to share. (\%20)

\section{Edit Username}

How to edit username in Facebook Messenger

1. In the Chats, click on my Facebook profile picture. (\%25)

2. Click on Username under the Profile section. (\%25)

3. Click on "Edit username". (\%25)

4. In the Username section, you can rewrite the already existing username. (\%25)

\section{Ignore Message}

How to ignore messages from someone (in messenger)

1. In the Chats screen, click on a recent conversation to reach someone's (either a friend or not) profile. (\%40)

2. Click on "i" (for info) icon at the top right. (\%10)

3. Close to the bottom click on "ignore messages" (\%40)

4. Press "Ignore". (\%10)

\section{Unignore Message}

How to unignore messages from someone

1. Click on People. (\%25)

2. On People section, press the "Add Contacts" icon at the top right corner. (\%25)

3. In Add controls, click on "Requests" with icon just below Add Contacts (one of the four icons right next to each other) (\%25)

4. In Message Requests, click "See all" to see filtered messages. (\%25)

\section{Activate Status}

How to activate Status.

1. In Chats, click on your profile picture. (\%25)

2. In Me section, click on "Activate Status" under Profile. (\%25)

3. In Activate Status, turn off Activate Status. (\%25)

4. Click on "Turn off" in the message box. (\%25) 


\section{RESULTS AND DISCUSSION}

Table 1 has given the successful performance of our participant on FB messenger in terms of total success point, time by indicating the knowledge of participant and, the number of steps for each function. If a participant knows how to do the task, the value is YES. As shown in Table 1, depending on the knowledge of VI, the duration time to complete tasks varied. Additionally, the successes of the tasks were dependent on the general behavior of the VI on SNS. In the Change Language task, the participant mixed the change language settings with the options for translating posts. That led her to waste considerable time. She achieved 100 points in change language task while spending the highest amount of time. The timing can be attributed to the highest number of total steps in this experiment. In the Change Profile Picture Task, there was no problem despite she did not want to do it. She did not want her friends to see her with a different picture. This change would easily be recognized by her friends as an update. She changed the picture back to normal as soon as she achieved it. Change Profile Picture was the second fastest after Activate Status and she received full points. Edit Username was the most problematic with 0 points and the longest time. That was mainly because no message showed the username was changed. She had already done in 1 minute 51 seconds. However, at that time, she could not understand that she made it. That was also because of the username she chose to change.

Table 1. Facebook Tasks' Performance in Terms of Points, Time, Knowledge, and Steps

\begin{tabular}{|c|c|c|c|c|}
\hline Function to Perform & Points & Time & Know-How to Do & Total Number of Steps \\
\hline Change Language & 100 & 3 minutes 15 seconds & Yes & 9 \\
\hline Change Profile Picture & 100 & 47 seconds & Yes & 4 \\
\hline Edit Username & 0 & 4 minutes & No & 4 \\
\hline Ignore Message & 0 & 1 minute 29 seconds & No & 4 \\
\hline Unignore Message & 25 & 2 minutes & No & 4 \\
\hline Activate Status & 100 & 25 seconds & Yes & \\
\hline
\end{tabular}

Normally (by default), the username is in the form of name.surname.number. However, she chose to make it in the form of namesurname.number. She could not understand it because of her lack of vision and message. Even for a sighted person, it might be difficult to do for the first time. She did not know about the operation so I told her how to do it. She was not familiar with either ignore or unignore message. I had to explain step by step. The problem about ignore was mainly because she confused it with block operation. She also could not understand why that operation is used. She received 0 points although she gave up the earliest in the ones that she had not known. For unignore operation, she correctly guessed that it is about the people menu but 4 icons on the request made her confused. Unignore message is related to the ignore message so she could get 25 points in 2 minutes. Activate Status was the easiest for her because that's the one she knew before. Moreover, she said that she used it regularly. Activate Status is the one with the best performance in terms of time (25 seconds) and one of the best for points (100 points).

\section{CONCLUSION}

In this paper, we make a VI usability analysis on Facebook in the Android platform. Six different Facebook functions are used to test the usability performance of VI on this site. Our results show that the knowledge of the VI about the task played a positive role in the task completion time. The best times are achieved when-knowledge about that specific function is concomitant with the high frequency of usage in the past. When a function includes lots of controls like unignore message, task completion time increases even it may not be completed by VI. If there is a similar function she remembered like in Language Settings, this might also be a problem. The most visible usability problem is the lack of a message in the edit username. A message like "Your username is changed" will make it much easier to understand. It will significantly reduce the time required to complete the task. As future work, we want to find more participants. Heuristic approaches may also be added to crosscheck the efficiency of the study. 


\section{ACKNOWLEDGMENT}

I would like to thank the Disability Unit of Bahcesehir University and Visually Impaired Association for their kind assistance.

\section{REFERENCES}

Aditya Vashistha, Edward Cutrell, Nicola Dell, et al. 2015. Social Media Platforms for Low-Income Blind People in India. In Proceedings of the 17th International ACM SIGACCESS Conference on Computers \& Accessibility. (pp. 259-272). New York, NY: ACM. Doi:10.1145/2700648.280985

Andre Rodrigues, Kyle Montague, Hugo Nicolau, et al. 2015. Getting Smartphones to Talkback: Understanding the Smartphone Adoption Process of Blind Users. In Proceedings of The 17th International ACM SIGACCESS Conference on Computers and Accessibility (ASSETS'15). October 26-28, 2015, Lisbon-Portugal. (pp.23-32).ACM. DOI: http://dx.doi.org/10.1145/2700648.2809842

Barbara Leporini, Maria Claudia Buzzi, and Marina Buzzi. 2012. Interacting with mobile devices via VoiceOver: Usability and accessibility issues. $O Z C H I^{\prime} 12,1-10$.

Bavani Ramayah, Azizah Jaafar, and Noor Faezah Mohd. Yatim. 2013. Visually Impaired User's Navigation Experiences in Facebook. IVIC 2013, LNCS 8237, 788-796.http://dx.doi.org/10.1007/978-3-319-02958-0_71

Berglind Smaradottir, Jarie Haland, Santiago Martinez. 2017. Accessibility of Mobile Devices for Visually Impaired Users: An Evaluation of The Screen-Reader VoiceOver. MEDINFO 2017: Precision Healthcare through Informatics. Doi:10.3233/978-1-61499-830-3-1381 p.1381

Brian M. Celusnak. September-October 2016. Teaching the iPhone with VoiceOver Accessibility to People with Visual Impairments. Journal of Visual Impairment \& Blindness.p.369-372

Brian Wentz and Jonathan Lazar. 2011. Are separate interfaces inherently unequal?: an evaluation with blind users of the usability of two interfaces for a social networking platform. In Proc iConference'11, 91-97.

Cynthia L. Bennett, Jane E, Martez E. Mott, et al. 2018. How Teens with Visual Impairments Take, Edit, and Share Photos on Social Media. In Proceedings of the SIGCHI Conference on Human Factors in Computing System (CHI'2018), Montreal, QC, Canada (p.1-11), https://doi.org/10.1145/3173574.3173650

Global social media ranking 2019. (n.d.). Retrieved June 19, 2019, from https://www.statista.com/statistics/272014/globalsocial-networks-ranked-by-number-of-users/

Haley MacLeod, Cynthia L. Bennett, Meredith Ringel Morris, et al. 2017. Understanding Blind People's Experiences with Computer Generated Captions of Social Media Images. In Proceedings of the SIGCHI Conference on Human Factors in Computing Systems (CHI' 2017), Denver, CO, USA, DOI: https://dx.doi.org/10.1145/3025453.3025814

Joao Guerreiro, Daniel Gonçalves. 2013. Blind People Interacting with Mobile Social Applications: Open Challenges. 3rd Workshop on Mobile Accessibility at the ACM SIGCHI Conference on Human Factors in Computing Systems.

Meredith Ringel Morris, Annuska Zolyomi, Catherine Yao, et al. 2016. "With Most of It Being Pictures Now, I Rarely Use It": Understanding Twitter's Evolving Accessibility to Blind Users. In Proceedings of the 2016 CHI Conference on Human Factors in Computing Systems (CHI '16), 5506-5516, https://doi.org/10.1145/2858116

Rakesh Babu, Rahul Singh. 2013. Accessibility and Usability of Social Media: Convergence between Blind Users and Design Standards. In Proceedings of the Nineteenth Americas Conference on Information Systems, Chicago, Illinois, August 15-17, 2013. p.1-9.

Rakesh Babu. 2014. Can Blind People Use Social Media Effectively? A Qualitative Field Study of Facebook Usability.American Journal of Information Systems, Vol. 2, 33-41.doi: 10.12691/ajis-2-2-2

Ramayah, B., Jaafar, A., N.F.M.: Accessibility Issues of Social Networking Sites to Blind Users in Malaysia. In: Visual Informatics International Seminar 2012 (VIIS 2012)(2012)

Shaomei Wu, Jeffrey Wieland, Omid Farivar, et al. 2017. Automatic Alt-text: Computer-generated Image Descriptions for Blind Users on a Social Network Service. In Proceedings of the 20th ACM Conference on Computer Supported Cooperative Work and Social Computing, https://doi.org/10.1145/2998181.2998364

Shaomei Wu, Lada A. Adamic. 2014. Visually Impaired Users on an Online Social Network. In Proceedings of the SIGCHI Conference on Human Factors in Computing Systems(CHI '14),3133-3142. New York, NY: ACM. https://doi.org/10.1145/2556288.2557415

Shi Qiu, Jun Hu, and Matthias Rauterberg. 2015. Mobile Social Media for the Blind: Preliminary Conference on Enabling Access for Persons with Visual Impairment (ICEAPVI), pp. 152-156.

Violeta Voykinska, Shiri Azenkot, Shaomei Wu, et al. 2016. How Blind People Interact with Visual Content on Social Networking Services. In Proceedings of the 19th ACM Conference on Computer-Supported Cooperative Work \& Social Computing (CSCW'16),1584-1595. https://doi.org/10.1145/2818048.2820013 